le portiQue Le Portique

Revue de philosophie et de sciences humaines

$19 \mid 2007$

Philosophies de l'argent

\title{
La pensée sapientielle de Pascal
}

De la recherche

Bernard M.-J. Grasset

\section{CpenEdition}

Journals

Édition électronique

URL : http://journals.openedition.org/leportique/1281

DOI : 10.4000/leportique.1281

ISSN : $1777-5280$

Éditeur

Association "Les Amis du Portique"

Édition imprimée

Date de publication : 1 janvier 2007

ISSN : 1283-8594

Référence électronique

Bernard M.-J. Grasset, « La pensée sapientielle de Pascal », Le Portique [En ligne], 19 | 2007, mis en

ligne le 15 décembre 2009, consulté le 25 mars 2021. URL : http://journals.openedition.org/leportique/ 1281 ; DOI : https://doi.org/10.4000/leportique.1281

Ce document a été généré automatiquement le 25 mars 2021.

Tous droits réservés 


\title{
La pensée sapientielle de Pascal
}

\author{
De la recherche
}

\author{
Bernard M.-J. Grasset
}

1 Avec Pascal, avec son Apologie de la religion chrétienne, nous découvrons au cœur du XVII siècle un témoignage particulièrement important d'une pensée à l'écoute de la Bible, une pensée qui s'inscrit dans l'héritage de la patristique et du Moyen Âge de l'intériorité, une pensée sapientielle. L'Apologie mêle indissolublement le travail de pensée et le travail d'exégèse. Pascal veut appeler l'homme à la recherche, à passer des ténèbres à la lumière. " [...] je ne puis approuver que ceux qui cherchent en gémissant " (fr. $421 \mathrm{~B} / 24 \mathrm{~S})^{1}$. La nécessité de chercher Dieu est un leitmotiv qui traverse toutes les Pensées, comme il traverse le pays biblique. Il n'est d'attitude juste pour l'homme embarqué, en situation d'exil, que de chercher, par-delà les opinions éphémères, la vérité. Le mot vérité devient ainsi un mot-clef du paysage apologétique ${ }^{2}$. Nous devons tendre de tout notre être, sans lassitude, à l'éclat du vrai. Cette recherche ardente de la vérité que Pascal recommande avec tant d'insistance à ses lecteurs, il en avait lui-même passionnément vécu ${ }^{3}$. Après l'avoir recherchée dans la science, il la découvrira dans la religion, là elle prend un Visage digne des plus hauts sacrifices. Rechercher la vérité revient à rechercher le salut. Seule la vérité peut apaiser l'angoisse de l'homme ${ }^{4}$. Mais elle demeure "couverte d'un voile", méconnue (fr. 843 B/425 S). La nature arrache l'homme à la lumière. La grâce l'incline au retour. Dans l'Apologie comme dans la Bible, la recherche de la vérité engage l'éternelle destinée de l'être humain et éclôt en sagesse.

1. De la Bible

2 Pascal est un homme de l'Écriture Sainte. Il «ne cesse, surtout après sa seconde conversion, de la lire et de l'étudier ${ }^{5}$. Avec l'expérience du Mémorial en novembre 1654 qui conféra un élan plus profond à la première conversion de Rouen en 1646, l'auteur de l'Apologie devient un lecteur fervent de la Bible. Il l'étudie, la médite et la prie. Le Mémorial est tissé de références bibliques. Les notes apologétiques qu'il commence de rédiger à partir de 1656 ne cessent d'interpréter l'écriture. Certains fragments des Pensées sont constitués de traductions bibliques, en particulier des prophètes $^{6}$. Des livres sapientiaux comme L'Ecclésiaste, Job aident à déchiffrer la 
condition humaine. Le projet apologétique gravite entièrement, et non seulement dans les liasses explicitement exégétiques, autour de l'Écriture. Pascal est " tout pénétré de la pensée biblique ${ }^{7}$. Tandis que la pensée de la Renaissance s'était éloignée du Dieu d'Abraham, du Dieu de l'Écriture, l'Apologie y voit le seul horizon de l'homme. L'importance accordée à la Bible était caractéristique des travaux des écrivains de PortRoyal mais Pascal lui donnera l'intense accent du témoignage.

3 L'influence des Saintes Écritures se traduira non seulement sur la pensée de l'apologiste mais aussi sur son style. Pascal puise ses images essentielles dans le texte sacré comme si leur origine transcendante leur conférait leur universalité. Il reprend de grands symboles bibliques tels la lumière et les ténèbres, le chemin, la terre et le ciel... ${ }^{8} \mathrm{Le}$ style des Pensées, imagé, symbolique est comme celui de l'Écriture, concret, vivant. De même que la langue hébraïque, il cultive la parataxe plutôt que l'hypotaxe. Certains fragments multiplient les coordinations avec et. Comme la Bible, Pascal aime l'hyperbole, les répétitions, l'inclusion et condense sa pensée en māshāl, en maxime, en proverbe. Il admire l'alliance de la simplicité et de la profondeur dans le texte sacré 9 . Le langage parabolique, symbolique, intuitif, proche des hommes devient le modèle à imiter. Sa prose, qui reprend volontiers le procédé sémitique du parallélisme, acquiert un véritable souffle poétique dans l'écho biblique. Le style des Pensées est, à l'image du style sacré, un style du cœur et du mystère ${ }^{10}$.

4 Pascal découvre dans la Bible une religion du sacrifice, du témoignage et de la rédemption. L'Évangile devient la norme d'une éthique de la charité. Il faut recentrer toute la vie sur l'absolu. En lecteur passionné de l'Écriture, l'auteur des Pensées conçoit l'existence comme un combat entre la nuit de la terre et la lumière du ciel, entre la chair et l'esprit. L'univers biblique est pour lui un univers de figures et le monde luimême est perçu comme une vaste figure de l'éternel. $\mathrm{Au}$ XVII ${ }^{\mathrm{e}}$ siècle, peu de temps après la mort de Pascal, commençaient à se dessiner les contours de l'exégèse moderne, historique et critique, notamment avec l'œuvre de l'oratorien Richard Simon. Se développe ainsi une approche scientifique, raisonnée de la lettre sacrée. Telle n'est pas la perspective de notre apologiste pour qui la lecture de la Bible n'est pas affaire de science mais de mystère. Le texte sacré luit comme un phare sur la liberté de l'être immergé dans le temps ${ }^{11}$. Sans la Bible l'homme égare sa vie dans le filet de l'erreur. Elle « n'est pas un livre comme les autres " ${ }^{12}$. La lumière qu'elle enseigne dépasse toute sagesse humaine. Pascal affirme ainsi des livres canoniques que "la vérité y est découverte ${ }^{13}$. Dans les épreuves du monde, la Bible demeure la clef qui préserve de l'abîme. Pascal trouve dans le Livre des livres la source de son interprétation du divin, de l'humain et de la nature ; une éthique, une esthétique, une eschatologie y prennent leur racine ; là se dévoilent en plénitude la sagesse et la vérité.

«Il faut [...] suivre les Pères et la tradition» (fr. 775 B/474 S). Pascal comprendra la Bible dans la continuité de la patristique et de la tradition spirituelle. Port-Royal se montrait particulièrement attaché aux études bibliques et patristiques ${ }^{14}$. Le Père qui a le plus influencé Port-Royal comme Pascal est saint Augustin. D'une manière plus générale, l'évêque d'Hippone a laissé une forte empreinte sur le xvII siècle religieux et spirituel. Après Augustin, il convient de souligner l'influence de saint Bernard, dont l'œuvre est elle-même marquée d'augustinisme. "Les religieuses de Port-Royal vénéraient saint Bernard comme le fondateur de leur ordre " ${ }^{15}$. Saint Augustin d'abord, l'abbé de Clairvaux ensuite demeurent les références patristiques majeures pour PortRoyal comme pour Pascal qui n'en éprouvait pas moins de l'admiration pour tous les 
Pères en général ${ }^{16}$. L'auteur des Pensées explore les deux sources de la vérité selon le catholicisme : les Saintes Écritures et la Tradition. Il faut revenir en matière de religion à « l'autorité de l'Écriture et des Pères " ${ }^{17}$. La règle de la tradition préserve de l'hérésie. La patristique représente aux yeux de Pascal le meilleur commentaire, le plus juste de la Bible. Si dans le domaine des sciences, il se montre résolument moderne, dans le domaine biblique, il reste fasciné par le passé. Bible et patristique, telles sont les véritables sources de sa pensée apologétique. Et qu'est-ce que le troisième ordre, cet ordre de la charité, de l'esprit (pneuma) et du mystère qui se situe au centre des Pensées, sinon la cristallisation de l'interprétation pascalienne de l'Écriture à la lueur de la patristique?

2. La sagesseA. Sagesse divine

6 Pascal combat la fausse sagesse, la sagesse profane qui veut définir le bien par ellemême, qui prétend se suffire. La sagesse sans Dieu ne peut porter de juste fruit, elle énonce le règne de la concupiscence. Il y a une forme d'orgueil, d'idolâtrie dans cette recherche de sagesse en dehors de l'absolu ${ }^{18}$. L'homme veut se déifier. À cette sagesse orpheline de la lumière s'oppose la sagesse de l'ailleurs, de l'infini, de l'éternité. La sagesse profane n'est qu'une sagesse naturelle, la sagesse sacrée est surnaturelle ${ }^{19}$. Inspiré par la Bible, Pascal conçoit cette autre sagesse comme une sagesse de l'amour. Ce sont les "yeux du cœur, qui voient la sagesse» (fr. 793 B/339 S). De tels yeux reconnaissent en elle des grandeurs «infiniment plus hautes» que celles des deux premiers ordres. Toujours dans ce même fragment, Pascal souligne que cette "grandeur de la sagesse [...] est invisible aux charnels et aux gens d'esprit " ${ }^{20}$. L'ordre de la chair, l'ordre de la raison ne pourront jamais atteindre la vraie sagesse. En concordance avec la Bible, Pascal pense la sagesse comme une marque du divin. Dieu « est sage » (fr. 654 B/802 S) ${ }^{21}$. Ce Dieu sage se présente comme un Dieu « puissant », un Dieu "véritable ». Sagesse et vérité se révèlent indissociables. Tout conflue vers la vérité divine, une vérité qui échappe au temps ${ }^{22}$. La Bible permet à l'homme de demeurer dans l'infinie sagesse. À vrai dire, Pascal ne condamne pas entièrement la sagesse profane. Même s'il y a une importante différence de nature entre celle-ci et la sagesse sacrée, certains éléments méritent d'y être puisés. Ainsi remarque-t-il à propos d'Épictète, représentant le stoïcisme, et de Montaigne, représentant le scepticisme, qu'ils « sont arrivés à quelque conformité avec la sagesse véritable qu'ils ont essayé de connaître ${ }^{23}$. Sceptique et stoïque approchent de la vérité mais uniquement de manière partielle : le premier voit la misère de l'homme en ignorant sa grandeur, le second voit la grandeur de l'homme en ignorant sa misère. Revenir à la Parole permet seul d'accéder à la plénitude de la sagesse. "Écoutez Dieu » (fr. 434 B/164 S). Tel est l'impératif qui conditionne toute la recherche sapientielle de l'Apologie. Sans écoute de la Voix, il ne peut y avoir que des fragments de sagesse. Écouter, c'est vivre son humaine condition dans l'orbe de la sagesse, de la vérité. L'acte d'écouter est synonyme, comme l'enseigne le lexique biblique, de l'acte d'obéissance ${ }^{24}$. "Aussi Dieu seul donne la sagesse » (fr. $460 \mathrm{~B} / 761 \mathrm{~S})^{25}$. La vraie sagesse relève de l'offrande, de la transcendance. L'écoute est la condition de la juste pensée. Pour "penser comme il faut ${ }^{26}$, l'homme doit, en se détachant du divertissement, des fuyantes apparences, penser à l'Être, à l'éternité. « Dieu doit régner sur tout et tout se rapporter à lui » (fr. $460 \mathrm{~B} / 761 \mathrm{~S})$. La sagesse pascalienne, à l'image de la sagesse biblique, demeure théocentrée.

7 Cette sagesse théocentrée se définit en même temps dans l'univers apologétique comme christocentrée. «Tout homme qui n'est pas sage de la sagesse de Jésus-Christ 
est fol $\gg{ }^{27}$. Cette affirmation du directeur spirituel de Port-Royal, Pascal la reprendrait volontiers à son compte. Sa pensée sapientielle est profondément orientée vers le Christ dans une lumière mystique. Des textes comme le Mémorial ou le Mystère de Jésus qui, s'ils n'appartiennent pas aux liasses apologétiques, n'en éclairent pas moins de l'intérieur leur cheminement, brûlent d'un ardent christocentrisme. Pascal est l'homme du tout, de l'absolu; ce tout, il l'a trouvé dans la personne du Christ, vrai visage de la sagesse. "Jésus-Christ est l'objet de tout et le centre où tout tend. Qui le connaît connaît la raison de toutes choses » (fr. 556 B/ 690 S). Sans le Christ, la pensée humaine ne peut discerner le dernier mot de la vie. Sans la présence christique, aucune sapience féconde ne peut advenir. Dans le Christ, «Homme-Dieu » où s'unissent ineffablement «deux natures ${ }^{28}$, se résolvent les antinomies des sagesses du monde, scepticisme et stoïcisme. Jésus est le nouvel Adam qui libère l'homme de la servitude du mal. À l'intérieur de l'homme, il constitue comme le portrait réimprimé du divin, que le monde avait effacé ${ }^{29}$. La venue du Libérateur a signifié pour l'humanité la découverte de la vérité en même temps que de la sagesse. «Il a fallu que la vérité soit venue, afin que l'homme ne véquît ${ }^{30}$ plus pour soi-même" (fr. $440 \mathrm{~B} / 497 \mathrm{~S}$ ). Celui qui selon l'enseignement néo-testamentaire est la Sagesse et la Vérité a épousé la condition humaine jusque dans sa plus grande déchirure. Ainsi «toute la souffrance humaine se trouve comme récapitulée en celle de l'Homme-Dieu. " ${ }^{31}$ La sagesse christique ne saurait être qu'une sagesse tragique. Jésus a souffert, Jésus est mort. Le passage par la mort révèle l'éternel amour du divin pour l'humain. « Jésus sera en agonie jusqu'à la fin du monde » (fr. 553 B/749 S). La sagesse de l'homme reviendra à mourir en communion avec celui qui ne cesse d'agoniser pour lui. La croix enseigne la sagesse de la conversion. Là, dans l'abandon du temps, le Christ a élevé l'humanité à son ultime vérité $^{32}$. Hors de la croix, la sagesse se referme sur le vide ${ }^{33}$. L'écriture apologétique tente de ramener le regard de l'homme des tourbillons du monde à la beauté sacrée du Crucifié. Comme la sagesse néo-testamentaire, la sagesse des Pensées est une sagesse de la croix.

\section{B. Sagesse et humanisme}

8 Pascal s'est passionné pour Dieu, il s'est aussi passionné pour l'homme ${ }^{34}$. Pour lui, le fonds de la condition humaine reste identique au long des siècles. Il y a simplement un avant la chute, un après la chute. En un sens, parce qu'elle met l'homme, sa situation existentielle, au cœur de sa pensée, l'Apologie aurait pu recevoir le titre De la condition humaine. L'étude des sciences, de la géométrie a laissé place à une étude plus essentielle: «l'étude de l'homme » ${ }^{35}$. Cette étude existentielle de l'homme prendra appui sur la Bible où l'apologiste voit sa condition dévoilée dans la vérité. Venant du plus lointain, de la sagesse scripturaire et de ses relectures patristiques, la conception pascalienne de la condition humaine se révèle très moderne, attentive aux blessures, à l'angoisse et à l'attente ineffaçable de l'être en exil. Au cœur de la sagesse apologétique, il y a le divin, il y a l'humain ${ }^{36}$. La sagesse des Pensées, théocentrée, christocentrée, est en même temps anthropocentrée. Ce qui traduit le mieux le mystère de l'humaine condition apparait comme l'oscillation entre misère et grandeur. Pascal a une conscience aiguë de la gravité de l'existence, traversée par la souffrance, hantée par cette mortalité que tous ont en partage. Avec Job, avec l'Ecclésiaste, il pense la misère tragique de la vie humaine ${ }^{37}$. L'Ecclésiaste enseigne la vanitas de l'existence, pleine d'illusions, éphémère séjour où aucun plaisir ne peut combler la soif d'absolu. «Nous travaillons incessamment à embellir et conserver notre être imaginaire, et négligeons le véritable» (fr. $147 \mathrm{~B} / 653 \mathrm{~S}$ ). Le néant, la vacuité, l'errance envahissent nos jours. 
Misère de l'homme. Misère du vide, misère aussi de cette souffrance incompréhensible dont Job, personnage symbolisant notre condition blessée, fait la tragique épreuve. Mais en même temps l'homme se détache par la pensée de tout le créé ${ }^{38}$. Contrairement aux autres créatures, il se sait mortel. Dans la souffrance il pense et peut se souvenir de son Créateur. "L'homme passe l'homme " ${ }^{39}$. - " [...] l'homme passe infiniment l'homme [...]» (fr. 434 B/164 S) Au-delà de l'enfermement terrestre, du gouffre de la ténèbre, vibrent en l'homme une trace du céleste, un rayon de lumière, une antique plénitude. «Dii estis » écrit Pascal, à la fin du même fragment, citant Ps 81,6 selon la Vulgate. Il y a une noblesse de l'homme, une liberté existentielle, une appartenance à l'ailleurs. Grandeur de l'homme, grandeur en lui de cette force de dépassement, de cette intériorité, dont l'importance fut soulignée par les prophètes et les Évangiles, où jaillit secrètement la flamme de la présence. En se retirant dans la solitude silencieuse de sa chambre, l'homme peut retrouver le chemin de la Parole. Pascal pense la misère et la grandeur de l'homme en interprétant l'Écriture et, en se souvenant à la lumière du texte sacré, de son expérience concrète de vie dans le monde, en société. Face à la tragique misère humaine, la sagesse pascalienne veut orienter les cœurs et les esprits vers l'absolu, la transcendance. Par-delà la vanité des apparences, la douleur de la finitude, la blessure du malheur, perdure intensément la nostalgie de l'être, de l'infini, du bonheur. S'il y a un humanisme dans les Pensées, c'est un humanisme de la lucidité, un humanisme qui sait l'homme familier du mal sans oublier pour autant son aspiration à la perfection, un humanisme biblique ${ }^{40}$. La sagesse des Pensées qui n'oublie pas toute la part d'ombre entourant la condition humaine cherche, dans l'ordre de la charité, à indiquer une issue. Éclairé par la Bible, l'humanisme apologétique est un humanisme des profondeurs, un humanisme qui prend une dimension spirituelle et mystique. L'anthropologie existentielle se dénoue dans une christologie. Tandis que l'athéisme reste enclos dans l'ordre de la chair ou l'ordre de la raison, la sagesse humaniste, entée sur la Bible, participe du troisième ordre, ordre du mystère, ordre christique.

3. Philosophie et penséeA. Pascal philosophe

9 La philosophie au XVII ${ }^{e}$ siècle a souvent une composition quadripartite où figurent la physique, la logique, la morale et la métaphysique. Le philosophe est aussi bien un savant et le domaine de la philosophie prend une vaste extension. Par rapport à la philosophie profane, Port-Royal adoptera, en la personne de Sacy, une attitude de rejet. En revanche Pascal est, comme Descartes, comme Leibniz, aussi bien philosophe que savant ${ }^{41}$. Certes il sait que ce n'est pas la réflexion philosophique qui apporte la vérité et rejoint Sacy dans la conviction d'une impuissance fondamentale de la philosophie, mais au lieu de la rejeter, il attend un désaveu de la philosophie et pense que l'échec de la philosophie présente en lui-même un intérêt ${ }^{42}$. Cette discussion autour de la philosophie avec Sacy trouvera dans l'Apologie de la religion chrétienne son accomplissement. La liasse X de l'édition Sellier (IX de l'édition Lafuma ${ }^{43}$ établie à partir de la première Copie) porte le titre de Philosophes. Le mot lui-même revient avec un réel indice de fréquence ${ }^{44}$. Des thématiques essentielles à la réflexion philosophique (nature, justice, raison, temps, bonheur, sagesse, beauté, vérité...) jalonnent le cheminement apologétique. Pascal est demeuré dans les profondeurs de son être en confrontation avec les philosophes comme en témoigne l'expérience du Mémorial. Sa culture philosophique n'est pas très vaste et se circonscrit avant tout à Platon ${ }^{45}$, Descartes son contemporain, Épictète et Montaigne. Mais ce qu'il cherche à atteindre à travers ces philosophes, c'est le cœur même de la philosophie. Platon, dans un monde 
polythéiste, a reconnu l'existence d'un Dieu unique, enseigné la vertu et affirmé l'immuabilité de l'âme, parente de l'éternel ${ }^{46}$. Descartes a su voir en la pensée la marque distinctive de l'humanité ${ }^{47}$. Quant à Épictète et Montaigne, figures emblématiques du stoïcisme et du scepticisme, ils ont su mettre en évidence la grandeur de l'homme pour le premier, sa misère pour le second. Dans le dogmatisme et le pyrrhonisme Pascal voit récapitulée toute l'histoire même de la philosophie. Au centre du débat philosophique se situe l'homme, sa bassesse et sa noblesse. Il y a une "sagesse de la terre " ${ }^{48}$. Les philosophes ont pu entrevoir la lumière. Au début du fr. 425 B/181 S (liasse XI Le souverain bien), Pascal reprend un constat philosophique universel : « Tous les hommes recherchent d'être heureux. » Le bonheur de l'homme en sa plénitude ne se trouve qu'en Dieu. "Lui seul est son véritable bien». Pascal est savant et philosophe mais aussi exégète. En passionné de la Bible, il verra le souverain bien de l'homme dans le Dieu révélé. De l'Entretien avec M. de Sacy aux Pensées, Pascal s'est intéressé à la philosophie, cherchant à saisir son essence même, et il a développé une véritable vision de l'univers et de la place de l'homme au sein de celui-ci. Il a réfléchi avec acuité sur la philosophie mais ne s'y est pas enfermé, sa foi ardente le guidant vers une autre contrée. Il a philosophé sur l'homme mais sans jamais cesser d'interpréter l'Écriture.

B. Au-delà de la philosophie

10 Pascal est philosophe et exégète. L'Apologie de la religion chrétienne se présente à la fois, de manière indissociable, comme une œuvre d'exégèse et une œuvre de pensée. De là naît l'unique beauté de ces fragments de témoignage. La pensée pascalienne est une pensée interprétative du texte sacré. Pour un penseur, se tourner avec ardeur vers la Bible signifie que la philosophie, incapable par elle-même d'atteindre ce qu'elle recherche, n'a pas d'autonomie absolue. Toujours en contrepoint de la raison se dessine le mystère.

11 Le constat philosophique essentiel de Pascal consiste à souligner l'échec de la philosophie: ni la sagesse, ni la vérité qu'elle prétend apporter ne s'y trouvent. Si Platon et Descartes ont eu des intuitions justes, ils n'ont jamais réussi à dépasser les limites de la raison pour découvrir une lumière à la fois surnaturelle et concrète. Le stoïcisme a méconnu la misère de l'homme, le pyrrhonisme en a négligé la grandeur. L'impasse de la philosophie vient de son enfermement dans le second ordre. La raison, la science, le savoir demeurent aveugles à la lumière de l'amour ${ }^{49}$. Il y a dans l'activité philosophique une volonté de savoir par soi-même qui s'exile de la grâce comme le geste de convoitise adamique au Jardin d'Éden. Les livres philosophiques forment un cercle qui ne peut avoir d'issue sans le Livre des livres. Il faut passer du discours désirant s'auto-suffire à la parole évangélique. La Bible devient la clef de l'aventure philosophique. Tandis que des philosophes comme Descartes, Leibniz, s'ils respectent l'Écriture Sainte, ne s'en servent pas pour leur réflexion philosophique ${ }^{50}$, chez Pascal sa lecture fervente permet de penser autrement, plus profondément, de s'élever du profane au sacré. L'auteur des Pensées avait une culture biblique extrêmement vaste ${ }^{51}$. La Bible est la lecture du troisième ordre, de l'ordre de la charité, de la conversion. Elle éclaire de son mystère les deux premiers ordres. Dans la perspective rationaliste, la philosophie explique la Bible; dans la perspective pascalienne, la Bible explique la philosophie. Au-delà de "la sagesse de la terre» demeure "la simplicité de l'Évangile " ${ }^{52}$. Choisir la Bible, ce n'est pas effacer la philosophie profane mais se situer en dehors d'elle pour en comprendre les richesses et les apories. Il faut lire Salomon, l'histoire de Job, il faut lire les livres sapientiaux, les Évangiles afin de penser 
l'existence humaine en sa vérité. Au sein du troisième ordre luit le phare de la Révélation. La vérité philosophique, la vérité de la raison appelle secrètement une vérité plus haute, la vérité biblique, la vérité du mystère.

Le langage apologétique, à l'image du langage biblique, traduit le concret, l'existentiel, le vécu. Pascal «n'aime guère les mots généraux abstraits, il leur préfère des termes précis répondant à des réalités " ${ }^{53}$. Les mots des Pensées sont les mots courants que leur usage biblique a sacralisés en les marquant du sceau de l'infini. Le langage abstrait, théorique, spécialisé, le langage philosophique, scientifique, technique n'atteint pas l'homme en son existence réelle. La pensée pascalienne ne cessera de réunir expérience et Révélation. Alors que le cartésianisme s'éloigne de l'expérience, l'apologiste y revient avec prédilection. Celui qui préfère l'Évangile à la philosophie peut accéder au troisième ordre. Là brille un Visage, un visage salvateur, libérateur, un Visage que ni le premier ordre, ni le second ordre ne connaissent, un Visage qui s'allie concrètement, jusqu'à l'agonie, avec l'existence humaine. Le point autour duquel gravite toute la pensée pascalienne se situe au-delà de la philosophie. Ni savant, ni philosophe ne peuvent répondre à la soif la plus essentielle de l'homme. Au centre du troisième ordre demeure le Christ $^{54}$. La pensée de Pascal apparait ainsi comme «une pensée entièrement déterminée par le Christ, inspirée directement par l'être du Christ. " ${ }^{55}$ Comme Augustin, Grégoire de Nazianze, les Pères en général, familier de l'Écriture il voit dans l'absence du Christ la principale imperfection de la philosophie profane. Le détachement lucide envers la philosophie permet un autre philosopher, un philosopher authentique ${ }^{56}$. Cet autre philosopher relie l'existence humaine à l'être christique. La vraie philosophie est une philosophie de l'amour. Les racines de la philosophie se trouvent dans la Bible.

C. De la raison au Dieu d'Abraham

13 Pascal, homme de science, géomètre, savait le pouvoir de la raison. Mais homme de foi, penseur, il en saisit les limites. La raison apparaît comme l'apanage de la philosophie du second ordre. " [...] nous ne nous devons jamais laisser persuader qu'à l'évidence de notre raison. ${ }^{57}$ La raison persuaderait efficacement l'homme. En se servant de la raison naturelle, celui-ci pourrait accéder à la sagesse et à la vérité. Pour Pascal au contraire, la raison n'est pas un instrument infaillible du vrai : « elle est ployable à tous sens. ${ }^{58}$ Influencée par la théologie janséniste qui accentue les conséquences du péché originel sur la condition humaine, Pascal pensera la raison comme viciée, altérée. Sa pensée en exagérera ainsi l'aveuglement, l'abaissement. En revanche l'auteur des Pensées discerne avec sagesse le juste équilibre entre le rationnel et le sur-rationnel. « Deux excès : exclure la raison, n'admettre que la raison » (fr. 253 B/214 S) (liasse XIV Soumission et usage de la raison, en quoi consiste le vrai christianisme). La raison ne doit pas prétendre légiférer sur tout, décider de tout -, ce ne serait que superbe. Outil utile et nécessaire, elle est appelée à s'incliner devant le mystère et à accomplir ainsi son ultime exigence. "La dernière démarche de la raison est de reconnaitre qu'il y a une infinité de choses qui la surpassent" (fr. $267 \mathrm{~B} / 220 \mathrm{~S}$ ) ${ }^{59}$. Ce qui se trouve en harmonie avec la raison, ce n'est pas de tout mesurer à l'aune de la raison mais de comprendre que tout ne peut se réduire à des lois rationnelles. La sagesse sait les limites de la raison. En contrepoint des philosophies de la raison, Pascal pense pour sa part qu'on ne peut rendre raison de tout. La vérité ne se réduit pas à tout ce qui est clair et distinct ; elle se couvre du voile du mystère. Des vérités les plus importantes, des vérités de la religion, l'homme est plus persuadé en son cœur que par le raisonnement ${ }^{60}$. La vraie philosophie accorde une place essentielle au cœur et au 
surnaturel. Plus haut que la froideur de la science, de la raison, parle la flamme du cœur, du sacré.

14 "Ils délibèrent de passer une heure ", écrit Pascal dans le fr. 219 B/505 S à propos des philosophes dont l'éthique ne s'inquiète pas de savoir si l'âme est immortelle ou non. Il faut ordonner la morale à l'éternité comme le souligne l'Écriture. Ce qui importe avant tout à l'existence humaine, c'est son destin eschatologique: soit l'enfer, soit le salut. «[...] nous n'estimons pas que toute la philosophie vaille une heure de peine » (fr. $79 \mathrm{~B} /$ 118 S) ${ }^{61}$. À quoi bon philosopher sur des points obscurs et incertains tandis qu'intérieurement son âme va à sa ruine ? La rédemption seule mérite la peine des jours de l'homme. Or cette rédemption, traduite sous le mode de l'intériorité, sous le mode poignant dans le Psautier, l'Évangile johannique, livres sacrés goûtés entre tous par l'apologiste, ne peut être apportée par les philosophes ${ }^{62}$. La philosophie ne mène pas au salut parce qu'elle ne mène pas au vrai Dieu. Le Dieu « des philosophes et des savants ${ }^{63}$ n'est pas le Dieu de feu, le Dieu rédempteur de la Bible. C'est un Dieu abstrait, un concept, une idée; il ne dialogue pas avec l'homme, n'entre pas intérieurement en alliance avec lui. «L'idée, dis-je, de cet être souverainement parfait et infini est entièrement vraie " ${ }^{64}$. À quoi bon une idée, même la plus élevée, quand tout dépend de la charité ? L'effort philosophique pour prouver Dieu se révèle infécond, inutile. "Les preuves de Dieu métaphysiques sont si éloignées du raisonnement des hommes et si impliquées, qu'elles frappent peu " (fr. $543 \mathrm{~B} / 222 \mathrm{~S}$ ) ${ }^{65}$. Ces preuves métaphysiques, héritées de la scolastique, ne rencontrent pas l'intériorité humaine. Pascal rejettera même la preuve de l'existence divine par la nature, ce en quoi il se montrera cette fois-ci en décalage avec la tradition biblique et patristique ${ }^{66}$. Le Dieu de la philosophie doit s'effacer devant le Dieu de la foi que l'on découvre à travers le Christ. La philosophie profane déchiffre rationnellement le monde comme un cycle régulier de causes et d'effets. Pour Descartes la lumière naturelle enseigne que tout a une cause de son existence. Dans cet univers de causes, Dieu devient la Cause par excellence, la cause ultime, «la cause de soi-même » ${ }^{67}$. Il est le grand horloger, la cause en quelque sorte mécanique des mouvements du réel, l'architecte du monde. À ce DieuCause, extérieur à l'homme, "auteur des vérités géométriques et de l'ordre des éléments ", Pascal préfère "le Dieu d'Abraham, le Dieu d'Isaac, le Dieu de Jacob» "Dieu d'amour et de consolation " ${ }^{68}$. Ce Dieu-là est un Dieu d'intériorité, qui touche le cœur, dont on peut témoigner dans les larmes et dans l'espérance. Le Dieu Causa sui, le Dieu-Idée n'est pas un Dieu que l'on peut aimer, prier, en présence duquel la pensée peut s'élever au lyrisme poétique, un Dieu du troisième ordre. Pour Pascal, « entre le Dieu des philosophes et le Dieu d'Abraham, Isaac et Jacob, la différence demeure insurmontable " ${ }^{69}$. L'expérience ardente du Mémorial lui avait révélé un Dieu de feu, un Dieu qui comble la soif humaine. Pascal a raison de dénoncer l'insuffisance du Dieu des philosophes, du Dieu du deuxième ordre, du Dieu simple Idée ou Cause. Un penseur autant nourri de la Bible qu'il l'était aspirait à un Dieu vivant. Mais il a durci à l'excès le cloisonnement entre les deux, négligeant la possibilité de passage, de transition entre le Dieu philosophique et le Dieu biblique, cette possibilité que s'étaient attachés le plus souvent à cultiver les Pères.

Pensée et religion

15 L'Apologie de la religion chrétienne est une œuvre de pensée où le profane se trouve constamment aimanté par le sacré. La pensée profane chemine vers la pensée religieuse. Autour de la pensée et de la religion se trace l'écriture apologétique ${ }^{70}$. La juste pensée exige l'accueil du dévoilement religieux. Sans la religion la pensée 
s'enferme dans les tourbillons des contradictions et l'errance de l'incomplétude. «La vraie philosophie [...] adopte comme principe de base la vérité de la religion chrétienne. ${ }^{71}$ La pensée chrétienne sera une pensée du mystère. En se conjoignant à la religion, la pensée s'édifie sur le mystère plus que sur la raison. La vraie pensée n'est pas contre la raison mais par-delà la raison; elle lit indéfiniment le texte sacré pour en restituer existentiellement l'écho. Le penseur religieux, étranger à la vanité du savoir éphémère, œuvrera dans la simplicité, l'humilité, illuminé d'une douce lumière intérieure. Dans son contact avec la Bible, il aura manqué à Pascal la rigueur historique et critique qui se développera avec Richard Simon. Mais il aura su traduire poétiquement sur le plan de la pensée l'essentiel du message biblique et ouvrir le regard sur une vivante Présence. S'il s'attache, à des fins apologétiques, à prouver géométriquement le christianisme, le plus juste de sa pensée religieuse relève de l'esprit de finesse. L'esprit de finesse donne à la réflexion philosophique de Pascal la profondeur de la pensée. L'esprit de finesse, vibrant d'intériorité, rassemble l'âme vers ce qui importe vraiment. Il n'est de féconde sagesse sans esprit de finesse. La pensée religieuse de Pascal tente de s'en tenir à la racine de l'être. «[...] sans l'Écriture, qui n'a que Jésus-Christ pour objet, nous ne connaissons rien [...] (fr. $548 \mathrm{~B} / 36 \mathrm{~S})$. Tourné vers l'essentiel, le sacré, Pascal devient « un penseur essentiel » ${ }^{72}$. Tandis que la pensée de Descartes est une pensée de l'oubli, la pensée de Pascal est une pensée de la mémoire. Chez le premier il s'agit de faire table rase du passé, de tout réédifier à partir d'un doute universel, chez le second il faut revenir à la Bible comme au fondement, et puiser dans la lointaine tradition patristique. À une pensée de l'expatriation s'oppose une pensée de l'enracinement.

4. Témoignage et FeuL'éclair du Mémorial

16 L'écriture pascalienne est une écriture de lutte et de témoignage ${ }^{73}$. Les fragments apologétiques combattent contre l'athéisme, en un témoignage authentique du mystère. Tout s'est cristallisé pour Pascal dans la nuit mystique du 23 novembre 1654. Désormais il sera par son existence et par l'œuvre des Pensées un témoin ardent du surnaturel. La lumineuse vérité d'un autre ordre de grandeur s'est révélée à son âme assoiffée. Un éclair d'amour a déchiré la nuit du monde ${ }^{74}$. Les citations bibliques dont le Mémorial est tissé reflètent intensément les mouvements du cœur de celui qui a été touché ineffablement par le mystère. La cohérence de son existence se jouera désormais dans le dialogue poignant avec le texte sacré et Celui qui en est, à ses yeux, la clef : Jésus-Christ ${ }^{75}$. Pascal est bouleversé à jamais. L'homme de la conversion devient un homme de témoignage. L'expérience du Mémorial transfigure Pascal en témoin de l'absolu. Le caractère profondément humain, vécu des fragments des Pensées résonnera comme un écho de cette expérience. En rencontrant le Témoin par excellence, Témoin de la vérité qui est passé par les affres de la souffrance et de la mort pour rédimer l'homme, bouleversé Pascal n'aura de cesse que de témoigner par sa vie et par son œuvre. Une flamme d'amour guide son chemin, une certitude l'habite ${ }^{76}$. Les Pensées nous touchent parce que, comme les textes bibliques, elles témoignent d'une expérience intérieure de la Présence. En même temps qu'il compose cette œuvre brûlante, le Pascal des dernières années, le Pascal de la conversion manifeste un amour intense, généreux pour les pauvres ${ }^{77}$. Écrire, exister dans l'orbe du mystère, blessé d'amour par l'éclair de l'ailleurs, c'est témoigner avec passion et vérité.

A. L'expérience du cœur 
17 Pascal a su discerner avec une rare acuité l'importance du cœur dans la Bible et l'a transposée dans sa propre pensée. Pour lui, «l'Écriture Sainte » est «la science du cœur ${ }^{78}$. La religion judéo-chrétienne se définit comme une religion $\mathrm{du}$ cœur. L'Apologie sera avant tout une apologie tournée vers le cœur ${ }^{79}$. Le cœur retrouvera dans les Pensées la riche signification qui est la sienne dans l'Écriture. Au cœur appartient ainsi une dimension cognitive, affective et éthique. Comme dans la Bible, le cœur pascalien est organe de connaissance, de pensée, de sagesse. En son cœur l'homme éprouve sentiments, passions, amour. C'est le cœur, sève cachée de la volonté, qui opère les choix décisifs de l'homme et peut être incliné par la main de Dieu ${ }^{80}$. Le cœur représente le secret, l'intériorité de l'être humain ; domaine du poignant, de la ferveur, de l'émotion, il intuitionne le vrai et relie en sa profondeur au sacré. Le cœur est la faculté de l'essentiel. Mieux que la raison, le cœur comprend le sens de la Bible. Plus qu'elle, il rapproche du divin. «C'est le cœur qui sent Dieu, et non la raison » (fr. 278 B/ 680 S). Le «Dieu sensible au cœur» est le Dieu des patriarches, des prophètes, des sages, du psalmiste, des apôtres et, selon l'expression d'Ac 9, 2, des fidèles de la Voie. Le cœur ouvre le regard humain sur l'infini, l'éternité, la vraie beauté. L'idéal serait de tout connaître par le cœur, de manière intuitive, immédiate. Tandis que la raison qui argumente, définit, déduit appartient à l'esprit de géométrie, le cœur qui expérimente intérieurement, intuitionne, saisit, appartient à l'esprit de finesse. Au raisonnement il faut toujours ajouter le sentiment ${ }^{81}$. Sans esprit de finesse, la vérité reste froide, distante, extérieure à l'homme. L'esprit de finesse au contraire rejoint la Présence. Incliné par la grâce, le cœur embrase d'amour pour le Crucifié.

Le domaine du cœur apparaît aussi bien comme le domaine de l'esprit. La connaissance par le cœur accède à la beauté d'une connaissance spirituelle. Le cœur qui croit, aime et espère détache l'homme de l'emprise du mal. Revenir au cœur, c'est trouver le chemin d'une spiritualité de l'humilité et de la charité. Le cœur est selon l'enseignement scripturaire le lieu de la conversion. Tout comme les Pensées sont une œuvre de cœur, le Pascal de la seconde conversion est un homme de cœur. Il se passionne pour les athées, ces pauvres de divin, et pour les miséreux. Accéder au troisième ordre, telle est la voie de la perfection. «On ne s'éloigne qu'en s'éloignant de la charité » (fr. 668 B/769 S). En une formulation poétique, Pascal rappelle l'homme à l'essentiel. L'expérience du cœur est une expérience du troisième ordre. Dans l'ordre de l'amour l'homme se trouve élevé au-delà de lui-même ${ }^{82}$. L'ordre surnaturel est un ordre où le croyant éprouve avec angoisse le tragique de l'amour infini qui a connu la souffrance et la mort, versant "telles gouttes de sang ${ }^{83}$ pour lui. L'homme de cœur vit spirituellement dans le troisième ordre. Les deux premiers ordres sont privés de cœur, de profondeur ${ }^{84}$. Le troisième ordre surpasse infiniment les autres ${ }^{85}$. L'ordre de l'amour luit intérieurement de sagesse et de sainteté. Le savant s'enferme avec sa raison dans le deuxième ordre, le sage, lui, témoigne avec son cœur dans le troisième ordre. L'expérience du cœur est une expérience de feu, d'embrasement. Il faut rechercher cet absolu qui murmure au plus secret de notre être son infinie charité ${ }^{86}$.

\section{B. L'expérience de l'esprit}

19 Dans l'Écriture Sainte, le chemin vers Dieu passe par l'intérieure conversion et revêt une dimension pénitentielle. La spiritualité de l'apologiste comme celle de Port-Royal et de Cîteaux se fondera sur l'exigence de la conversion et la nécessité de l'ascèse. Homme de la Bible, Pascal est aussi un homme spirituel et son œuvre en porte la trace incandescente ${ }^{87}$. Il aimait méditer, prier ; sa piété, nourrie de la Bible, du Psautier, des 
Évangiles, inscrit sa vie dans la profondeur. Prier revient à imiter le Christ. " Il [Jésus] prie. ${ }^{88}$ À l'image de l'agonisant du Jardin des Oliviers, le fidèle doit prier jusqu'à l'extrême. La prière ouvre l'esprit de l'homme sur la Présence ${ }^{89}$. Devant le mystère du Deus absconditus, la souffrance du solitaire de Gethsémani, la prière est appelée à se prolonger indéfiniment. "[...] prions plus longtemps.» écrit Pascal à la fin de sa méditation sur l'épisode évangélique du Jardin des Oliviers (fr. 553 B/749 S). Chez l'apologiste, comme chez les Pères, la pensée n'est jamais éloignée de la prière. La Bible elle-même doit être interprétée avec un esprit d'orant. L'Apologie de la religion chrétienne trouvera son élan spirituel dans la conjonction d'une pensée priante et d'une prière pensante. Prière et pensée s'épousent en écho d'une expérience de l'esprit. Aux sources des Pensées, il n'y a pas seulement des textes mais un souffle intérieur venu du sacré.

L'être de l'homme a pour Pascal une dimension spirituelle essentielle. Le troisième ordre, l'ordre surnaturel, l'ordre du cœur est un ordre de l'esprit. S'élever au troisième ordre revient à devenir veilleur. "Jésus sera en agonie jusqu'à la fin du monde. Il ne faut pas dormir pendant ce temps-là " (fr. $553 \mathrm{~B} / 749 \mathrm{~S}$ ). Veiller, telle est la juste attitude de l'esprit. Le Mystère de Jésus, qui représente à nos yeux comme la conclusion secrète des Pensées, invite à une sagesse de veilleur. Dans les épreuves tragiques de la condition humaine, dormir reviendrait à laisser triompher les ténèbres. Les Pensées au contraire veulent susciter un homme de la veille, qui allume une lampe et se tient à l'écoute de la Parole. La sagesse de veilleur est une sagesse biblique, évangélique ${ }^{90}$. Il faut se tenir en silence, à l'écart des bruits qui font oublier l'essentiel, et écouter, écouter la voix lointaine de l'ailleurs. L'expérience de l'esprit comprend la temporalité dans le mystère de l'éternité ${ }^{91}$. Cette sagesse de veilleur apparaît aussi bien comme une sagesse de l'enfance. "La Sagesse nous envoie à l'enfance» (fr. 271 B/116 S) ${ }^{92}$. La sagesse de l'enfance brille humblement comme une sagesse de l'esprit ${ }^{93}$. L'esprit d'enfance, mis en avant par l'Écriture, purifie le regard et sait reconnaître l'amour du divin. L'expérience de l'esprit oriente vers la beauté, la paix et la vérité. L'esprit élève l'existence à un amour universel, y compris de l'ennemi. Le vieil homme, l'homme de péché dont parle saint Paul, doit se transformer intérieurement, devenir un homme nouveau afin de chanter sans fin le « cantique nouveau », « ce chant qui part de l'esprit nouveau de la charité ${ }^{94}$.

C. L'expérience du mystère

21 L'esprit de finesse aspire à tout "voir [...] d'un seul regard » ${ }^{95}$, sans intermédiaire, à expérimenter en une vraie sagesse la présence même. Cette aspiration à une vision pleine, entière, concrète, totale, c'est dans le mysticisme que Pascal la réalisera. L'être qui pourra combler parfaitement la vue humaine ne peut être que Dieu. L'homme marche en exil dans un univers nocturne mais «si Dieu lui découvre quelques rayons de son essence ${ }^{96}$, il peut connaître et aimer en vérité. Le Dieu de Pascal est tout à la fois un Dieu voilé et dévoilé. Il se voile dans la nature, l'Écriture, l'Incarnation et l'Eucharistie ${ }^{97}$. Dieu caché, Dieu qui se cache, mais aussi Dieu révélé, Dieu qui se révèle. Il est « caché en partie, et découvert en partie » (fr. $586 \mathrm{~B} / 690 \mathrm{~S}$ ) ${ }^{98}$. La vérité advient à l'homme comme à travers une nuée. Voilement et dévoilement se mêlent en un "avènement de douceur ", selon la belle expression du fr. 430 B/182 S. La condition humaine est expérience du mystère. Ce mystère, par-delà la raison, est lumineux; il rend compréhensible ${ }^{99}$. Lorsque l'expérience du mystère devient intérieure rencontre, embrasement du cœur et élévation de l'esprit au seuil de la Présence, elle prend la forme de l'expérience mystique. La beauté secrète des Pensées vient de leur contact ardent avec le mystère. Un élan mystique guide la parole fragmentaire. Les trois textes 
les plus mystiques de Pascal dessinent l'architecture voilée de l'Apologie. Le Mémorial constitue le prélude, le fragment sur les trois ordres le centre et le Mystère de Jésus l'épilogue. Ces trois textes, pleins d'émotion, de ferveur, sont christocentrés et témoignent d'une ineffaçable rencontre. Une lecture des Pensées qui ignorerait et le Mémorial et le Mystère de Jésus laisserait s'effacer l'essentiel. Ces deux textes vibrent d'un mysticisme totalement imprégné des Saintes Écritures ${ }^{100}$. La ferveur christique, mystique du Mémorial et du Mystère de Jésus, nous la retrouvons dans le fr. 793 B/339 S sur les trois ordres. Le troisième ordre est un ordre de mystère, un ordre mystique. L'absolu est Présence - Shekhīnāh, Parousia, Praesentia. "Je te suis présent " confie le Christ à Pascal dans le dialogue mystique du Mystère de Jésus. Cette présence mystérieuse se découvre comme une présence d'amour, d'amitié. «Je te suis plus ami » poursuit ce dialogue. Devant une telle présence aimante, une si mystérieuse amitié, il n'est d'attitude juste que de s'abandonner, s'offrir entièrement ${ }^{101}$. L'abandon spirituel apparaît comme la condition de l'union mystique. Il y a eu chez Pascal une volonté forte d'union, de communion ${ }^{102}$. L'union mystique, la communion spirituelle, loin de l'éclatement du monde, rend l'homme librement participant de la plénitude divine, restaure l'unité perdue. "Adhaerens Deo unus spiritus est ${ }^{103}$. [...] Tout est un. L'un est en l'autre. Comme les trois Personnes » (fr. 483 B/404 S).

Le troisième ordre est ordre du cœur, de l'esprit et du mystère. Dans un monde obscur, l'homme doit toujours chercher les signes de la présence, reconnaître la voix du mystère. Un Dieu qui se voile et se dévoile ne s'impose pas mais s'offre doucement à la liberté de l'homme. Au cœur de l'interprétation pascalienne de l'Écriture, il y a un Dieu caché, un Verbe voilé. L'apologétique des Pensées prend sa source dans un mysticisme biblique. Le combat contre l'athéisme naît d'une passion mystique. Le lyrisme mystique représente le véritable horizon des Pensées. Pascal est incontestablement de Port-Royal par sa théologie mais par son mysticisme ardent, son témoignage bouleversant, il est au-delà, il n'est plus de Port-Royal. Son expérience du mystère a culminé dans la mystique. Ami de la sagesse, de la beauté et de la vérité, interprète de l'Écriture, Pascal fut à la fois, comme le souligne Jean Steinmann, "philosophe», "poète» et « mystique ${ }^{104}$.

Poésie et vérité

Pascal s'émerveillait de la beauté et de la vérité du style biblique, un style rempli de charité ${ }^{105}$. Ce style lui servira de référence pour son écriture qui saura avec bonheur allier pensée et poésie. Le langage obscur, technique, complexe, sans beauté de l'expression, lui reste étranger ${ }^{106}$. La recherche de la sagesse et de la vérité chez l'auteur des Pensées, qui s'intériorise en recherche spirituelle et mystique, est aussi bien une recherche esthétique. À l'image de l'Écriture il faut unir le beau, le bien et le vrai. L'expérience mystique du Mémorial qui est à la source des Pensées sera retranscrite sous le mode poétique. Le langage du Mémorial frappe par son sobre lyrisme, son intensité méditative, spirituelle - une intention plastique préside à la disposition des mots en versets, entrecoupés de silences symbolisés par des traits horizontaux, surmontés et suivis dans la copie du parchemin d'une croix entourée de rayons ${ }^{107}$. Le lyrisme plein d'émotion du Mémorial se retrouvera dans les Pensées, en particulier dans les fragments les plus spirituels et mystiques, qui apparaissent également comme les plus poétiques. Le chant intérieur transfigure le tragique de l'existence en beauté. Tandis que le style des Provinciales reste de nature classique, le style des Pensées, imprégné de la Bible, éclôt en poème en prose. L'art apologétique est un art de la métaphore et du refrain, qui, refusant un usage neutre de la langue, attentif au choix des mots et de leur ordre, 
s'attache avant tout à émouvoir. Par son goût de l'expressivité, de la simple suggestion, de la densité, de l'éclair, du raccourci, de la brièveté, du fragment, le style concis et fulgurant des Pensées, inspiré de la Bible, annonce la poésie moderne dont Pascal doit être considéré comme l'un des précurseurs secrets ${ }^{108}$. Familier de l'ellipse, il cultive une poétique de l'essentiel. Un texte comme Sépulcre de Jésus-Christ (fr. $552 \mathrm{~B} / 467 \mathrm{~S}$ ) où les phrases sont disposées comme des vers introduits par un titre, apparait par sa sobriété, son dépouillement et son intensité un véritable joyau poétique. La poésie des Pensées jaillit de la Bible et du Christ qui en est le centre. Interprétant dans la lumière augustinienne le beau Psaume 137, d'une poignante nostalgie, Chant de l'exilé, le fr. 459 B/748 S, qui commence ainsi: "Les fleuves de Babylone coulent, et tombent et entraînent ", vibre tout entier d'une ardente flamme poétique. Le souffle du poème, venu du plus lointain, confère sa musique si particulière au langage des Pensées. La pensée ne s'organise pas selon la froideur du concept mais atteint le lecteur à travers une mélodie de feu, une mélodie tragique. Pascal, si méfiant à l'égard de l'art, des fausses beautés, est devenu, en imitant la Bible où beauté et vérité s'unissent, un " grand artiste en langage » ${ }^{109}$. Sans poésie, jamais la philosophie ne pourra atteindre le cœur des hommes. L'aphorisme pascalien conjugue poème et sagesse. Une philosophie sans aphorismes développe une pensée sans poésie. La pensée poétique se cristallise en éclats de vérité.

Pascal dans les Pensées est non seulement penseur et exégète, mais aussi poète. La pensée revêt ainsi une dimension esthétique, poétique. Le « cœur nouveau » appelle un "langage nouveau " ${ }^{110}$. Le langage nouveau, le langage du cœur circoncis est le langage $\mathrm{du}$ troisième ordre. La philosophie rationnelle parle le langage du deuxième ordre, un langage technique, conceptuel, sans poésie; la philosophie biblique parle l'autre langage, un langage du cœur, de l'esprit et du mystère. Le travail pascalien sur le langage ne saurait se dissocier d'une recherche spirituelle et mystique en même temps que d'une éthique de la charité. L'ordre du cœur est aussi l'ordre de la poésie. Il n'est d'autre lieu que le troisième ordre où philosophie et poésie peuvent se rassembler dans l'écho scintillant de la sagesse, de la beauté et de la vérité.

Conclusion

25 Comme celle des Pères, la philosophie de Pascal se trouve en harmonie avec la Bible. L'amour, la spiritualité et le mystère y tiennent une place essentielle. La sagesse, la beauté et la vérité naissent $\mathrm{du}$ sol biblique et prennent le visage du Christ. L'existentialisme pascalien s'origine dans l'existentialisme sacré. La Bible a permis à l'auteur de l'Apologie de la religion chrétienne de dépasser le scepticisme et de comprendre l'essence même de la philosophie. Des versets-clefs ont contribué à façonner la vision anthropologique, éthique, philosophique, théologique, sotériologique, eschatologique du penseur-exégète ${ }^{111}$. La pensée, exprimée dans un souffle de lyrisme contenu, traduit une expérience intérieure de l'absolu. Sans la Bible, Pascal n'aurait pas fait œuvre de pensée. Avec un singulier relief, en lecteur profond du texte sacré, il a développé l'importance du cœur dans l'existence humaine. C'est là sans doute son plus grand apport à une lecture philosophique, pensante de l'Écriture. En revanche, en établissant une "distance infinie", un "écart radical» entre les deuxième et troisième ordres ${ }^{112}$, la raison et la charité, le savoir et le mystère, le Dieu des philosophes et le Dieu d'Abraham, Pascal se situe en décalage par rapport à la tradition patristique qui cherchait plutôt à concilier, réunir, à établir des passages entre les deux. La position de l'apologiste est en cela plus extrême. Mais l'ensemble de l'Apologie n'en constitue pas moins un merveilleux témoignage d'une pensée, qui est 
aussi bien une sagesse, enracinée avec force et justesse dans la Bible, et restant fidèle dans ses orientations essentielles aux Pères ainsi qu'à leurs continuateurs médiévaux. Pascal apparaît ainsi comme le dernier grand témoin, au sein du christianisme, d'une philosophie bâtie sur l'Écriture, en pleine consonance avec elle. Les Pensées se déploient tout entières comme une œuvre de philosophie exégétique, d'exégèse philosophique. Puisant ainsi dans les sources lointaines, elles mettent en œuvre une pensée sapientielle, une pensée qui ne dissocie pas la sagesse et l'expérience, la vérité et le révélé, une pensée du cœur qui aime à se dire en poème.

L'importance de la sagesse dans les Pensées est trop souvent ignorée. C'est pourtant elle qui rayonne au sein du troisième ordre, c'est elle que le fragment central de l'Apologie (793 B/339 S) évoque avec force. Tournée vers la recherche de la vérité, la pensée pascalienne est aussi une quête de la sagesse. Elle puise dans les textes sapientiaux de l'Ancien comme du Nouveau Testament afin de déchiffrer la condition humaine, son angoisse, sa blessure ${ }^{113}$. Elle s'exprime volontiers dans des maximes proches du māshāl hébraïque, dans un style symbolique, parabolique. La pensée sapientielle de Pascal est une pensée existentielle, une pensée du témoignage. Reliée au sacré, centrée sur le divin et l'humain, cette pensée prend une connotation spirituelle. Cultivant l'esprit de finesse, elle se tient à l'écoute du mystère. Le penseur devient un veilleur qui trouve dans la croix le sommet de la sagesse. Il n'est de sagesse sans charité, sans inclination du cœur. La pensée de Pascal reste aimantée en son fond par la sagesse. La pensée voisine avec la beauté, la philosophie avec la poésie. La pensée sapientielle de Pascal est une pensée de la mémoire, une pensée du cœur et du mystère, une pensée poétique. Nourrie de la Parole, le langage de la sagesse et de la vérité devient langage de beauté.

\section{NOTES}

1.. L'abréviation fr. est utilisée pour fragment, B renvoie à l'édition Brunschvicg des Pensées et opuscules, Paris, Classiques Hachette, 1978, (désormais abrégée en Bm); S à l'édition Sellier (établie à partir de la Seconde Copie), Pensées de Pascal, Paris, Bordas, Classiques Garnier, 1991.

2.. Avec 204 occurrences, le mot vérité est l'un des plus fréquents des Pensées. (On trouve également 30 occurrences au pluriel). (L'adjectif vrai apparaît quant à lui à 105 reprises). Voir Hugh M. DAVIDson, Pierre H. DUBÉ, A Concordance to Pascal's Pensées, Cornell University Press, 1975, p. $1461 ; 1464$.

3.. Adressée après la mort de Blaise à M. Périer, son beau-frère, la Lettre de M. d'Andilly évoque « son extrême amour pour la vérité, qui lui était [...] beaucoup plus chère que sa vie », CEuvres complètes, éd. Jean Mesnard, Paris, Desclée de Brouwer, 1964-1992, t. IV, p. 1530 (Nous abrégerons la référence à cette édition sous la forme O.C.).

4.. «[...] il n'y a de consolation qu'en la vérité seule », Lettre sur la mort de son père, 17 octobre 1651, Bm, p. 97.

5.. André GOUNELLE, La Bible selon Pascal, Paris, PUF, 1970, p. 8.

6.. Voir les liasses LIV Prophéties [1], LVI Prophéties [3] et LIX Prophéties [6] de l'édition Sellier. 
7.. Ernest JOVY, Études pascaliennes, t. VIII, Les Antécédents de «l'infiniment petit » dans Pascal, Paris, Vrin, 1932, p. 161.

8.. On sait par ailleurs le rôle décisif joué par le grand symbole biblique du feu dans le Mémorial.

9.. "Jésus-Christ a dit les choses grandes [...] simplement » (fr. 797 B/340 S).

10.. Au sujet du style des Pensées, nous nous permettons de renvoyer à notre essai Les Pensées de Pascal, une interprétation de l'Écriture, Paris, Kimé, 2003, p. 270-277.

11.. « L'Écriture a donc pour Pascal une importance capitale. Elle est la parole de Dieu qui ouvre à l'homme le secret de sa destinée et lui montre le chemin du salut. » (André GOUNELLE, op. cit., p. 26).

12.. Ibid., p. 21.

13.. Lettre sur la mort de son père, Bm, p. 97.

14.. " La science patristique de Saint-Cyran était considérable », Mgr Bernard JACQUELINE, « Les milieux jansénistes français au XVII ${ }^{\mathrm{e}}$ siècle et saint Bernard », Cîteaux 6, 1955 , p. 28. Port-Royal s'est engagé non seulement dans la traduction du texte sacré (Bible de Sacy) mais aussi dans celle des Pères (Antoine Arnauld et Robert Arnauld d'Andilly ont traduit des livres d'Augustin, Le Maistre de Sacy a traduit Jean Chrysostome).

15.. Mgr Bernard JACQUELINE, "L'influence de saint Bernard au XVII ${ }^{\mathrm{e}}$ siècle ", Collectanea Cisterciensia, t. 42, 1980/1, p. 29. Au-delà de Port-Royal, l'auteur de cet article souligne "l'influence très considérable de saint Bernard au XVII ${ }^{\mathrm{e}}$ siècle ». Ses œuvres sont traduites, « les biographies se succèdent » (Ibid., p. 22 ; 35). (Sur l'influence bernardine, voir également du même auteur : « Les milieux jansénistes français au XVII ${ }^{\mathrm{e}}$ siècle et saint Bernard », p. 29-30).

16.. Augustin est le Père de loin le plus cité ou évoqué dans les Pensées (fr. $186 \mathrm{~B} / 490 \mathrm{~S}$; 193 B/657 S ; 234 B/480 S ; 270 B/205 S ; 283 B/329 S ; 543 B/222 S ; 654 B/802 S ; 655 B/ 315 S ; 812 B/200 S ; 869 B/452 S...). Saint Bernard est cité dans les fr. 549 B/224 S ; 551 B/246 S ; 654 B/802 S ; 920 B/746 S... Outre ces Pères de prédilection, l'œuvre de Pascal aime se référer aux Pères (Irénée, Hilaire de Poitiers, Jérôme...) comme à autant d'autorités.

17.. Préface sur le Traité du vide, O.C., t. II, p. 779.

18.. " Dans la sagesse, l'orgueil proprement. " - « Le lieu propre à la superbe est la sagesse » (fr. 460 B/761 S). Ce fragment esquisse la conception des trois ordres qui prendra toute sa force dans le fr. $793 \mathrm{~B} / 339 \mathrm{~S}$. Il est symptomatique de constater que dans ces deux fragments autour des trois ordres, le mot sagesse occupe une place essentielle, comme s'il constituait une véritable clef de voûte de l'univers apologétique. 19.. Pascal conclut le fr. 793 B/339 S, qui représente vraiment le cœur des Pensées, en qualifiant le troisième ordre, celui de l'authentique sagesse, de « surnaturel ». 20.. Il faut entendre ici le mot esprit, comme souvent dans l'Apologie, en son sens classique d'intelligence, d'entendement, de raison. Le deuxième ordre est l'ordre de la raison et de la science : comme symbole du deuxième ordre, Pascal cite le personnage d'Archimède.

21.. À rapprocher d'Is 31,2: « Il [Dieu] est sage », ou encore de Si 1,8 et Rm 16,27 (Nous citerons l'Écriture selon la traduction de la Bible de Jérusalem, adoptant les abréviations et conventions en usage).

22.. À la fin de la $12^{\mathrm{e}}$ Provinciale, Pascal note : « la vérité subsiste éternellement ", citant le Ps 116,2 (" hê alêtheia tou kuriou ménei eis ton aiôna. »). (Les Provinciales, éd. L. Cognet, Paris, Bordas, Classiques Garnier, 1992, p. 235). 
23.. Entretien avec M. de Sacy, éd. P. Mengotti et J. Mesnard, Paris, Desclée de Brouwer, 1994, p. 122.

24.. Le verbe shāmā' en hébreu a le double sens d'écouter et d'obéir, double sens que l'on retrouve dans le akouô grec.

25.. On retrouve ici un écho pascalien de Pr 2,6: «Car c'est le Seigneur qui donne la sagesse » (« hoti Kurios didôsin sophian » - « quia Dominus dat sapientiam »). (Ni J. LHERMET dans Pascal et la Bible, Paris, Vrin, 1931 (tableau statistique des citations bibliques, p. 186-194), ni Ph. SELLIER dans son édition des Pensées et dans Pascal et la liturgie, Genève, Slatkine, 1998 (index des citations bibliques, p. 117-141), ni E. MARTINEAU dans Discours sur la religion, Fayard/Armand Colin, 1992 (table biblique, p. 273-279) n'ont identifié ce rapprochement). Dans le fr. 793 B/339 S sur les trois ordres, Pascal souligne que « La grandeur de la sagesse [...] est nulle sinon de Dieu ».

26.. Fr. $146 \mathrm{~B} / 513 \mathrm{~S}$.

27. SAINT-CYRAN, Écrits de piété inédits, in Jean ORCIBAL, La Spiritualité de Saint-Cyran, Paris, Vrin, 1962, p. 261.

28. Entretien avec M. de Sacy, p. 126.

29.. Prière pour demander à Dieu le bon usage des maladies, BM, IV, p. 59.

30.. Forme du subjonctif imparfait en usage au XVII ${ }^{\mathrm{e}}$ siècle à côté de la forme vécût qui l'a supplantée.

31.. André FEUILLET, L'Agonie de Gethsémani. Enquête exégétique et théologique suivie d'une étude du « Mystère de Jésus » de Pascal, Paris, Gabalda, 1977, p. 258.

32.. « En Jésus-Christ, Pascal voit l'accomplissement et le salut de la condition humaine douloureuse et rachetée, la figure bouleversante de l'homme. Sur le visage de Jésus-

Christ en agonie, il discerne comme le reflet du visage humain misérable et splendide. » (Lucien JERPHAGNON, Pascal et la souffrance, Paris, Éditions ouvrières, 1956, p. 171).

33.. " En rien fors qu'en la Croix je ne cherche de gloire, / Aussi bien sans la Croix tout le monde n'est rien. » Lazare de SELVE, Les Euvres spirituelles sur les Évangiles des jours de Caresme et sur les Festes de l'année, Genève, Droz, 1983, Sonnet LVI, p. 95. Ces vers, publiés pour la première fois en 1620, de Lazare de Selve, poète qui a été inspiré par l'Évangile et a su heureusement allier sagesse, spiritualité, sens du mystère et sobre pureté du langage, auront comme un écho lointain dans l'ardente prose christique des Pensées. 34.. Les deux mots les plus employés dans les Pensées sont Dieu et homme, à l'image du lexique biblique où Dieu et homme sont également les termes les plus utilisés (homme étant simplement précédé de fils et de roi dont la fréquence s'explique par l'usage hébraïque de préciser l'identité en notant la filiation et par l'importance historique et messianique de la royauté). (Voir notre essai, p. 276).

35.. Fr. 144 B/566 S. Le regard purement scientifique empêche le contact vivant, intérieur avec l'humanité dont il faut sans cesse penser les caractéristiques concrètes. Vincent Carraud parlera à propos de Pascal d'« anthropologie phénoménologique ", d'« analytique existentiale » (Pascal et la philosophie, Paris, PUF, 1992, p. 453).

36.. On notera que les mots sage et sagesse appartiennent au vocabulaire récurrent des Pensées : la Concordance de H. M. Davidson et P. H. Dubé identifie 30 occurrences de sagesse, 26 de sages et 15 de sage.

37.. «Salomon et Job ont le mieux connu et le mieux parlé de la misère de l'homme " (fr. 174 B/22 S) (Avec l'exégèse traditionnelle, Pascal croyait que Salomon était l'auteur de Qohélet).

38.. « Toute notre dignité consiste donc en la pensée. » (fr. 347 B/232 S).

39.. Texte rayé par Pascal et correspondant à une première rédaction du passage. 
40.. François Mauriac écrit à juste titre : «Pascal : le seul humaniste digne de ce beau nom ; le seul qui ne renie rien de l'homme; il traverse tout l'homme pour atteindre Dieu » (Blaise Pascal et sa sœur Jacqueline, Paris, Hachette, 1931, p. 251).

41.. On sait l'œuvre scientifique de l'inventeur de la machine arithmétique, ses expériences sur le vide, ses traités mathématiques. On peut lire dans l'Entretien avec $M$. de Sacy : « Il [Sacy] crut donc devoir ainsi mettre M. Pascal sur son fort ${ }^{\mathrm{a}}$, et lui parler des lectures de philosophes ${ }^{b}$ dont il s'occupait le plus. » (P. 92) (a : fonds, Bm, p. 148 ; b : philosophie, ibid.). Le Pascal d'après le Mémorial, tourné vers la mystique, donne une part de son temps aux lectures et aux études philosophiques.

42. André GOUNELLE, L'Entretien de Pascal avec M. de Sacy, Étude et commentaire, Paris, PUF, 1966 , p. $127 ; 129$.

43.. Paris, Seuil, L'Intégrale, 1963.

44.. La Concordance de H. M. Davidson, P. H. Dubé donne 33 occurrences pour philosophes. Le mot philosophe quant à lui apparaît à 9 reprises.

45.. « Platon, pour disposer au christianisme » (fr. 219 B/505 S). Par cette affirmation, Pascal se montre l'héritier de la patristique. Sa connaissance du philosophe athénien doit sans doute d'ailleurs beaucoup à Augustin.

46.. " [...] l'âme est immortelle [athanaton] », Phédon, 73 a (Paris, Garnier Flammarion, 1965, p. 123). (Voir aussi 114 d, p. 176).

47.. « Mais je ne puis concevoir l'homme sans pensée », écrit Pascal dans le fr. 339 B/ $143 \mathrm{~S}$, en écho du Discours de la méthode (IV ${ }^{\mathrm{e}}$ partie).

48.. Fr. 435 B/240 S.

49. La philosophie, « cantonnée » dans l'ordre de l'intellect, « ne saurait voir l'ordre de la charité, visible aux seuls yeux de l'amour. » (Gilles ouıvo, " Pascal. Apologétique et philosophie : bref état de la question ", XVII siècle, $n^{\circ}$ 185, Octobre-Décembre 1994, p. 735).

50.. Voir Xavier tilliette, Les Philosophes lisent la Bible, Paris, Cerf, 2001, p. 12.

51.. Sa sœur Gilberte Périer souligne qu' « il la [l'Écriture Sainte] savait toute par cœur » (Vie de Blaise Pascal, Bm, p. 16).

52.. Fr. $435 \mathrm{~B} / 240 \mathrm{~S}$.

53.. Fortunat STROWSKI, Les Pensées de Pascal. Étude et analyse, Paris, Mellotée, 1948, p. 225. «Une particularité de la langue de Pascal est justement la faculté de rendre concrètes les généralités ", Jean-Jacques DEMOREST, Pascal écrivain. Étude sur les variantes de ses écrits, Paris, Éditions de Minuit, 1957, p. 101.

54.. Dans le fr. 793 B/339 S, en contrepoint de l'ordre d'Archimède voué au savoir, s'élève l'ordre de Jésus-Christ voué à la sainteté.

55.. Xavier tilliette, Le Christ de la philosophie, Paris, Cerf, 1990, p. 36.

56.. «Se moquer de la philosophie, c'est vraiment philosopher » (fr. 4 B/671 S).

57.. DESCARTES, Discours de la méthode, Paris, Vrin, 1999, IV partie, p. 100. « [...] la raison est un instrument universel [...] » Ibid., $\mathrm{V}^{\mathrm{e}}$ partie, p. 121. Il faut user de « la lumière de la raison ». LEIBNIZ, Opuscules philosophiques choisis, Paris, Vrin, 1978, « Remarques sur la Partie générale des Principes de Descartes », p. 59. La raison permet de rendre raison de tout. « [...] rien ne se fait sans la plus haute raison », Ibid., p. 78.

58.. Fr. 274 B/455 S. Voir également fr. 82 B/78 S : « Plaisante raison qu'un vent manie, et à tout sens ! » L'agir humain est soumis à des forces souterraines. "L'homme n'agit point par la raison, qui fait son être » (fr. 439 B/736 S). La raison a été faussée par la chute. Il y a une réelle « corruption de la raison ». (fr. 440 B/497 S). 
59.. Voir aussi fr. 272 B/213 S : « Il n'y a rien de si conforme à la raison que ce désaveu de la raison $»$.

60. Voir fr. 278 B/680 S ; 279 B/487 S.

61. Fragment écrit en référence à Descartes et rayé par Pascal.

62.. «[...] Le salut est hors de portée de la philosophie. » André BORD, Pascal et Jean de la Croix, Paris, Beauchesne, 1987, p. 210.

63.. Mémorial, O. C., t. III, p. 50.

64.. DESCARTES, Méditations métaphysiques, Paris, PUF, 1974, Méd. III, p. 70. Voir également ibid., p. 61 où est évoquée l'idée (idea) par laquelle est conçue « un Dieu souverain, éternel, infini, immuable ». Dieu est une idée née (ingenita) avec moi. Ibid., Méd. V, p. 103. Dans le Discours de la méthode, Descartes parle de « l'idée d'un être plus parfait que le [s]ien », de « l'idée » qu'il avait « d'un être parfait ». (IV e partie, p. 92 ; 95-96). 65.. Voir aussi fr. $556 \mathrm{~B} / 690 \mathrm{~S}$ : « je n'entreprendrai pas ici de prouver par des raisons naturelles, ou l'existence de Dieu, ou la Trinité, ou l'immortalité de l'âme, ni aucune des choses de cette nature ".

66.. « Eh quoi ! Ne dites-vous pas vous-même que le ciel et les oiseaux prouvent Dieu?» - Non » (fr. 244 B/38 S). Prouver Dieu par « le cours de la lune et des planètes » ne sert de rien (fr. 242 B/644 S). Sans doute faut-il voir ici la marque de l'influence janséniste qui accentue l'état de corruption de la nature alors même que le Psautier exalte sa beauté (« Les cieux racontent la gloire de Dieu » (Ps 19,2) ; « la terre est remplie de ta [Seigneur] richesse » (Ps 104,24)) et la sagesse sacrée y reconnaît un signe de la Présence (« la grandeur et la beauté des créatures font, par analogie, contempler leur Auteur » (Sg 13,5)).

67.. Méditations métaphysiques, 1 ères Réponses, p. 142-143. Voir aussi LEIBNIZ : « la raison dernière des choses est au-delà du monde, elle est Dieu » (Op. cit., « De la Production originelle des choses ", p. 84). Dieu, cause de lui-même, est la cause originelle de toutes les causes dérivées. Il faut « rechercher les causes efficientes de toutes les choses qui sont au monde, sans en excepter Dieu même " (Méditations métaphysiques, $4{ }^{\text {es }}$ Réponses, p. 214). En cela ces philosophes à la recherche des causes se montrent les héritiers de la scolastique, et avec le thomisme ils pensent le divin comme unité de l'existence et de l'essence. « [...] je ne saurais concevoir autre chose que Dieu seul, à l'essence de laquelle l'existence appartienne avec nécessité » (DESCARTES, Méditations métaphysiques, Méd. V, p. 104) ; «[...] il existe un Être unique, métaphysiquement nécessaire, c'est-à-dire dont l'essence implique l'existence [...] » (LEIBNIZ, op. cit., p. 84).

68.. Fr. 556 B/690 S.

69.. Paul RICÆUR, « De l'interprétation à la traduction », in André LACOCQUE, Paul RICCEUR, Penser la Bible, Paris, Seuil, 1998, p. 363.

70.. Cette importance est soulignée sur le plan lexical. La Concordance Davidson - Dubé donne ainsi 37 occurrences de pensée et 23 de pensées, 33 de penser (plus 19 de pense, 11 de pensent, 9 de pensons, 6 de pensé, pensera), 161 de religion et 21 de religions.

71.. Thomas MORE HARRINGTON, «Stratégie et philosophie dans les Pensées de Pascal », Cahiers d'histoire des littératures romanes, 1/2, 2002, p. 194. Voir également ibid., p. 190 : «Seules la vraie religion et la vraie philosophie possèdent cette solution-là [Chute et Rédempteur Homme - Dieu], qui "passe le dogmatisme et le pyrrhonisme et toute la philosophie humaine" (L 131 - S 164), y compris la sagesse de la terre ».

72.. Expression de Charles PÉGUY pour qui les Pensées appartenaient aux rares « œuvres essentielles ». Euvres en prose complètes, Paris, Gallimard, Bibliothèque de la Pléiade, 
1988, II (1905-1909), De la situation faite à l'histoire et à la sociologie dans les temps modernes, VIII-3 (4.11.1906), p. 496.

73.. L'auteur des Pensées « n'est pas un docteur, mais un écrivain de combat », André GOUNELLE, La Bible selon Pascal, p. 6. (Voir également du même auteur, L'Entretien de Pascal avec M. de Sacy. Étude et commentaire, p. 124).

74.. De « ce FEU du Mémorial où se surimpriment les figures bibliques et convergent les souvenirs majeurs d'une existence », " Pascal [...] gard[era] toujours le témoignage », Pierre MAGNARD, « Le principe de similitude », Méthodes chez Pascal, Paris, PUF, 1979, p. 408.

75.. Le mot Jésus-Christ revient à quatre reprises dans le texte du Mémorial comme un refrain de lyrisme spirituel et mystique.

76.. Après le miracle de la Sainte Épine (24 mars 1656), Pascal change de cachet, ne voulant plus que « celui qu'il se fit graver, et qui représentait un ciel ${ }^{a}$ renfermé dans une couronne d'épines, avec ces mots de saint Paul : Scio cui credidi ${ }^{\mathrm{b}}$ ", Mémoires de FONTAINE, O. C., t. I, p. 957 (a. Variante : œil ; b. 2 Tm 1,12 : « je sais en qui j'ai mis ma foi »).

77.. Dans sa Lettre à M. de Saint-Calais (3 septembre 1662), NICoLE souligne qu'« il a fait de grandes charités durant sa maladie et presque tout donné son bien à sa mort. » ( 0 . C., t. IV, p. 1541).

78.. Gilberte PÉRIER, Vie de Blaise Pascal, Bm, p. 16.

79.. Avec, selon la Concordance de la Bible de Jérusalem (Paris, Cerf, Brepols, 1982), 1024 occurrences, le mot cœur se situe au rang 18 des substantifs les plus employés dans la Bible. La Concordance des Pensées, avec 124 occurrences, place le mot cœur au rang 14 des substantifs (Le mot charité apparaît pour sa part à 37 reprises et le mot amour à 32 reprises dans l'Apologie).

80.. Sur l'inclination du cœur en lien avec Ps 119,36, voir fr. 284 B/412 S ; 287 B/414 S. 81.. « Nous connaissons la vérité, non seulement par la raison, mais encore par le cœur » (fr. 282 B/142 S).

82.. Ainsi « l'ordre de la charité est celui de la grâce et de la vie surnaturelle » (André FEUILLET, Le Christ Sagesse de Dieu d'après les Épîtres pauliniennes, Paris, Gabalda, 1966, p. 383).

83.. Fr. $553 \mathrm{~B} / 751 \mathrm{~S}$.

84.. «L'ordre de la charité est de s'enraciner dans le cœur » (fr. 772 S - manuscrit Joly de Fleury).

85.. Dans son étude sur « Le thème des trois ordres dans l'organisation des Pensées », Jean MESNARD écrit : « Dès lors, c'est à une confrontation du premier ordre et des deux autres, mais avec subordination du second au troisième, que nous assistons souvent dans la partie proprement religieuse des Pensées. Au couple charnel/spirituel répondent beaucoup d'autres : lettre/esprit (219/251)a figurant/figuré (270/670), visible/invisible (275/643), nature/grâce (ibid.), extérieur/intérieur (219/251), circoncision du corps/ circoncision du cœur (270/670) » (a. La première référence chiffrée renvoie à l'édition Lafuma, la seconde à l'édition Brunschvicg), (La Culture du XVII siècle, Paris, PUF, 1992, p. 476). Mais spirituel, esprit, figuré, invisible, grâce, intérieur, circoncision du cœur ne constituent-ils pas des termes caractéristiques, en dernier ressort, du troisième ordre $^{b}$ ? Ainsi pensons-nous que la dichotomie se situe entre les deux premiers ordres d'une part qui relèvent d'une façon générale de la lettre, de la chair, de la concupiscence et le troisième ordre d'autre part qui relève de l'esprit (spiritus), de la charité, du mystère. L'anthropologie pascalienne retrouve la conception paulinienne de 
l'existence comme combat de l'esprit contre la chair, particulièrement accentuée dans l'augustinisme. (b. Si le mot esprit a ordinairement dans les Pensées le sens classique de mens, d'entendement (voir p. 123, n. 20) et appartient ainsi au deuxième ordre, il reçoit aussi, en référence à la Bible et de manière essentielle, celui de spiritus, souffle de l'âme et devient par là propre au troisième ordre. Quand Pascal oppose la lettre à l'esprit, c'est bien avant tout au sens de spiritus dans l'héritage de saint Paul (voir 2 Co 3,6 et notre essai, p. 35-36) qu'il pense, au troisième ordre).

86.. « Mon Dieu n'est rien qu'amour » écrit, reprenant sous le mode du lyrisme intérieur l'affirmation de $1 \mathrm{Jn} 4,8.16$, Claude HOPIL, auteur plein de ferveur mystique et dont les vers, qui aspirent à contempler l'Être, manifestent une rare élévation de pensée (Les Divins Élancements d'amour, Cantique XI, in Jean ROUSSET, « Un poète théologien et mystique du XVII e siècle ", Nova et Vetera, XXXII, $n^{\circ} 1,1957$, p. 276). 87.. Parmi les œuvres de Pascal empreintes de spiritualité, citons sa correspondance (Lettre sur la mort de son père, Lettres à Mlle de Roannez...), des opuscules (Prière pour demander à Dieu le bon usage des maladies, Sur la conversion du pécheur...), des textes mystiques comme le Mémorial ou le Mystère de Jésus, et bien sûr aussi les Pensées. 88.. Abrégé de la vie de Jésus-Christ, 211, O. C., t. III, p. 290. Voir également le Mystère de Jésus évoquant le même épisode de Gethsémani : « Jésus prie »(fr. 553 B/749 S). 89.. « Prions Dieu de nous le faire reconnaître et servir en tout », Lettre IV à Mlle de Roannez (fin octobre 1656), Bm, p. 215 ; O. C., t. III, p. 1037.

90.. Voir Ps 130,6 ; Mt 25,13...

91.. « Éternellement en joie pour un jour d'exercice sur la terre », Mémorial (O. C., t. III, p. 51).

92.. Pascal cite à la suite Mt 18,3 selon la Vulgate.

93.. Une telle sagesse habite la pensée et la vie de Pascal. Les témoins de la fin de sa vie comme M. de Sainte-Marthe ou le Père Beurrier, ont souligné qu'il quittait le monde avec la simplicité, l'humilité d'un enfant (Vie de Blaise Pascal, Bm, p. 34-35; version longue, éd. Lafuma, p. 31).

94.. Lettre V à Mlle de Roannez (5 novembre 1656), Bm, p. 216 ; O. C., t. III, p. 1038.

95.. Fr. $1 \mathrm{~B} / 670 \mathrm{~S}$.

96.. Fr. $430 \mathrm{~B} / 182 \mathrm{~S}$.

97.. Sur le Deus absconditus (expression empruntée par Pascal à Is 45,15 selon la Vulgate), voir fr. 194 B/681 S, 242 B/644 S, 518 B/752 S, 556 B/690 S, 585 B/275 S, 751 B/ $260 \mathrm{~S}$, ainsi que la Lettre IV à Mlle de Roannez. Ce sentiment de l'occultation du divin se rencontre aussi chez les poètes spirituels et mystiques du XvII siècle. «Soleil qui fus voilé en l'incarnation, / Éclipsé en ta mort, et en ta passion » Lazare de SELVE, op. cit., Sonnet XXXI, p. 70 ; « Le Soleil est caché » Claude HoPIL, Les Divins élancements d'amour, Cantique XI, in Jean RousseT, art. cit., p. 275.

98.. « Au lieu de vous plaindre de ce que Dieu s'est caché, vous lui rendrez grâces de ce qu'il s'est tant découvert » (fr. 288 B/13 S). Voir aussi fr. 557 B/690 S ; 559 B/690 S ; 848 B/690 S.

99.. Voir fr. $434 \mathrm{~B} / 164 \mathrm{~S}$ à propos du mystère du péché originel.

100.. André FEUILLET, exégète de métier, met en avant à juste titre « la familiarité extraordinaire avec la Bible attestée par le Mystère de Jésus et le Mémorial », L'Agonie de Gethsémani, p. 289.

101.. " Renonciation totale et douce. » Mémorial ; " Seigneur, je vous donne tout », Mystère de Jésus. 
102.. Avant que de mourir, tout son être n'a d'autre volonté que de recevoir l'Eucharistie. «[...] il demanda avec des instances incroyables qu'on le fît communier [...] », Vie de Blaise Pascal, Bm, p. 38.

103.. 1 Co 6, 17 : «Celui qui s'unit au Seigneur n'est avec lui qu'un seul esprit ».

L'ensemble du fragment s'inspire de Paul, en particulier de la comparaison de l'Église au corps (1 Co 12,12-30).

104.. Les Trois Nuits de Pascal, Paris, Desclée de Brouwer, 1962, p. 9.

105.. « Le style de l'Évangile est admirable en tant de manières, et entre autres en ne mettant jamais aucune invective contre les bourreaux et ennemis de Jésus-Christ » (fr. $798 \mathrm{~B} / 658 \mathrm{~S})$.

106.. L'Apologie « concili[e] la poésie avec un langage clair, [...] évitant le jargon des théologiens et des philosophes » (Jean-Jacques DEMOREST, op. cit., p. 136).

107.. «Le Mémorial constitue, s'il en est, le " poème illumination », tout entier tendu vers l'expression de l'indicible ", Olivier Jousus, « Pascal poète en prose ", XVII siècle, $\mathrm{n}^{\circ} 221$, Octobre 2003, p. 745. Autour du mot FEU (en lettres capitales dans la copie) se déroule le récit poétique de l'expérience de l'indicible.

108.. Au sujet du caractère poétique de la langue apologétique, en lien avec la Bible, voir notre essai p. 284-292.

109.. Sully PRUDHOMme, La Vraie Religion selon Pascal, Paris, Félix Alcan, 1905, p. 20. Il a ainsi témoigné d'une « souveraine maîtrise dans l'art d'écrire » (Ibid., p. 375). La force si juste de son style biblique explique qu'un auteur comme Paul Claudel en ait souligné la rare beauté, alors même qu'il se sentait par ailleurs étranger à sa théologie.

110.. Lettre V à Mlle de Roannez, Bm, p. 216 ; O. C., t. III, p. 1038. Et Pascal de citer à l'appui $\mathrm{du}$ langage nouveau Mc 16,17 (L'expression cœur nouveau est d'origine prophétique. Voir Ez 36, 26).

111.. Parmi ces versets-clefs, on peut citer Ex 3,6 sur le Dieu d'Abraham, Is 45,15 sur le Deus absconditus, Is 53, 3-4.7 sur le Serviteur souffrant et silencieux, Ez 36, 26 sur le renouvellement du cœur et de l'esprit, le passage du cœur de pierre au cœur de chair, Ps 82, 6 sur la grandeur humaine, Ps 119, 36 sur l'inclination du cœur, Qo 1, 2 sur la misère humaine, la vanitas, Mt 22, 36-40 sur les deux grands commandements de l'amour du divin et de l'humain a , Lc 10, 42 sur l'unique nécessaire, Jn 8, 36 sur la vraie liberté, Jn 17, 3 sur le lien entre vie éternelle et connaissance de Dieu et de son Envoyé, 1 Co 1, 21-25 sur l'aveuglement de la sagesse du monde et l'autre sagesse, la sagesse de la croix, la sagesse divine, $\mathrm{Ph}$ 2, 8 sur la kénose, l'obéissance et la croix, Ph 2, 12 sur le salut dans la crainte et le tremblement... (a. : Cf. fr. 484 B/408 S : « Deux lois suffisent pour régler toute la République chrétienne, mieux que toutes les lois politiques »; fr. 670 B/301 S: «L'unique objet de l'Écriture est la charité »).

112.. Vincent CARRAUD, op. cit., p. $384 ; 457$.

113.. Comme à la fausse beauté qui n'est qu'ornement éphémère s'oppose la vraie beauté qui relie à l'éternité, comme au faux amour qui n'est que concupiscence, amourpropre s'oppose la caritas, amour vrai qui ouvre à la lumière, à la fausse sagesse qui n'est qu'orgueil s'oppose la vraie sagesse qui accueille la Parole. 


\section{RÉSUMÉS}

Passionné de vérité, Pascal est aussi en quête de sagesse. Il trouve dans la Bible la source d'une pensée du cœur, attentive au mystère, qui donne sa place à l'inquiétude spirituelle. La vraie sagesse naît de l'écoute de la Parole. Si elle est utile, la sagesse profane demeure impuissante à saisir la plénitude de la vérité. La pensée sapientielle de Pascal, de nature existentielle, tragique, se centre sur le Christ qui illumine secrètement l'humaine condition. Exégétique, cette pensée trouve une issue à l'impasse philosophique dans le séjour à l'intérieur du texte sacré. La sagesse d'inspiration biblique ordonne la raison à l'amour. La pensée sapientielle de Pascal ne peut se dissocier de l'expérience incandescente du Mémorial. C'est une pensée du témoignage, vibrante d'un humanisme lucide et poignant. Sans juste langage il n'est de véritable sagesse. La pensée sapientielle de l'auteur de l'Apologie de la religion chrétienne prend l'inoubliable coloration du poème.

\section{AUTEUR}

\section{BERNARD M.-J. GRASSET}

Bernard M.-J. Grasset est docteur en philosophie. En 2003, il a publié un essai issu de sa thèse, Les Pensées de Pascal, une interprétation de l'Écriture, aux éditions Kimé. Traducteur de l'hébreu, il a publié de nombreux recueils de poésie ainsi que, notamment à l'étranger, des articles de recherche gravitant autour de la Bible et de la philosophie. 